\title{
Geosimulation as a Tool for the Prevention of Traffic Accidents
}

\section{Autores}

Amelec Viloria, Noel Varela, Luis Ortiz-Ospino, Omar Bonerge Pineda Lezama

\begin{abstract}
Traffic accidents represent a never-ending tragedy, and according to the World Health Organization (2018), 1.33 million people die in the world every year [1]. Most efforts in modeling phenomena of a dynamic nature have focused on working with static snapshots that reduce the natural depth of the world's dynamics to simplify it, abstracting perspectives that are fixed or static in some way. In the case of traffic accidents, most models used are those based on the principle of cause and effect, where the appearance of one or several variables gives rise to the event, like a domino effect. In this research, the problem of traffic accident avoidance was addressed through the use of a dynamic type model, based on the technique called geosimulation, where all the elements involved are interrelated.
\end{abstract}

Palabras clave

Traffic accidents, Geosimulation, Agent-based modeling, Geographic information systems, Dynamic models, Traffix 